\section{Confounding Variables}

James H. Baños

Department of Medical Education, University of Alabama School of Medicine, Birmingham, AL, USA

\section{Synonyms}

Confound; Extraneous variable; Lurking variable

\section{Definition}

A confounding variable is an extraneous variable that obscures the true relation between two other variables or groups of interest. In experimental research designs, a confounding variable often presents as an unintended or undesirable systematic difference between groups (the independent variable) that is also systematically related to the outcome of interest (dependent variable). It hinders the ability to infer a causal relation between the variables and can lead to misattributing a causal effect to the independent variable (a threat to internal validity). Potential confounding variables are most effectively addressed during study design (e.g., via random assignment, case control matching), but may be addressed to some extent during statistical analysis (e.g., handled as a covariate).

\section{Cross-References}

Analysis of Covariance (ANCOVA)

- Error, Sources of

\section{References and Readings}

Kazdin, A. E. (Ed.). (2016). Methodological issues \& strategies in clinical research (4th ed.). Washington, DC: American Psychological Association.

Pearl, J. (2009). Causality: Models, reasoning, and inference (2nd ed.). Cambridge: Cambridge University Press. 